\title{
Ultrasound of the hand in systemic sclerosis
}

\author{
Horatiu Ioan Popov ${ }^{1,2}$, Maria Magdalena Tamas ${ }^{1,3}$, Cristina Pamfil ${ }^{1,3}$, Simona Rednic ${ }^{1,3}$ \\ 1"Iuliu Hatieganu" University of Medicine and Pharmacy, Cluj-Napoca, Romania \\ ${ }^{2}$ Department of Rheumatology, Emergency County Hospital, Zalau, Romania \\ ${ }^{3}$ Department of Rheumatology, Emergency County Clinical Hospital, Cluj-Napoca, Romania
}

\begin{abstract}
Systemic sclerosis is a chronic connective tissue disease characterized by multi-organ involvement but the main clinical changes occur in the hands, secondary to skin, joint and microvascular damage. Therefore the hand received a special attention for imaging and especially for ultrasound evaluation. In rheumatology US become an extension of the clinical examination and particularly in systemic sclerosis it has been proven to help with a better assessment of the skin, blood vessels, joints and tendons involvement. This evolution was allowed by permanent improvement of technology along with expanding the range of ultrasound applications which happened especially in past decade.
\end{abstract}

Keywords: systemic sclerosis, ultrasound, hand, musculoskeletal

\section{BACKGROUND}

Systemic sclerosis (SSc) is a chronic connective tissue disease characterized by multi-organ involvement and may affect the skin, vessels, the heart, joints, kidneys and lungs (1). Ultrasound (US) is a cost-effective, noninvasive, accessible imaging modality that clinicians use at the point of care to assess disease activity, morphostructural changes, and therapeutic efficacy regarding different rheumatic conditions. Therefore, US is available for assessment of different organ targets involved in SSc (2).

\section{MUSCULOSKELETAL}

In rheumatology and especially in rheumatology related-US, it is obvious that the musculoskeletal (MSK) system receives the most attention and SSc is a connective tissue disease with frequent MSK involvement, reported between 46 and 97\% $(3,4)$. MSK involvement could include stiffness, arthritis, tendon sheath involvement, joint contractures, and proximal muscle weakness, but hands (particularly the metacarpophalangeal $[\mathrm{MCP}]$ and proximal inter- phalangeal [PIP] joints), and wrists are the most commonly affected joints (5). Data from the European League Against Rheumatism Scleroderma Trial and Research Group (European Scleroderma and Trials and Research group) database indicate point prevalences of $16 \%$ for synovitis, $11 \%$ for tendon friction rubs, and $31 \%$ for joint contractures (6). It is well known that US is superior to clinical examination in detecting wrist and hand synovitis and tenosynovitis in SSc $(7,8)$. Very recently, a new study reconfirmed the previous publishe data (9).

\section{Joints}

In order of frequency, the main changes detected by US of the hands and wrists joints in SSc pateints are: joint effusion, synovial proliferation associated or not with power doppler (PD) signal, periarticular calcinosis and joint space narrowing. Joint effusion and synovial proliferation are found in half of cases with significantly higher and more complex involvement of the wrist $(7,10)$. A high prevalence of synovial hypertrophy and joint effusion was demonstrated in SSc patients with arthralgia, with an $11-49 \%$ rate 


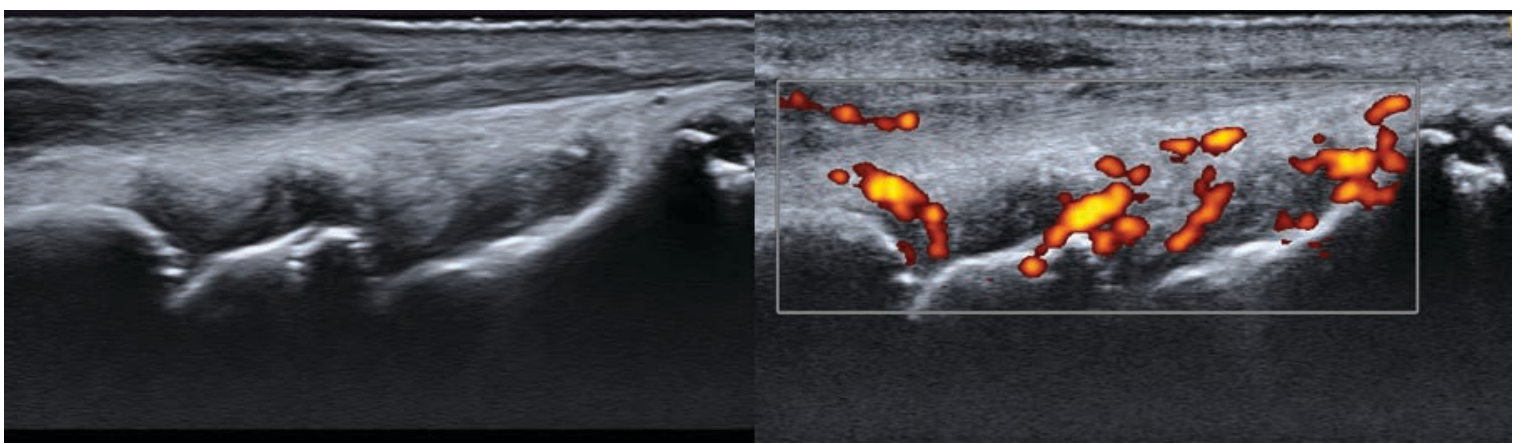

FIGURE 1. Dorsal longitudinal scan of the wrist with evidence of synovial hipertrophy with intense PD signal
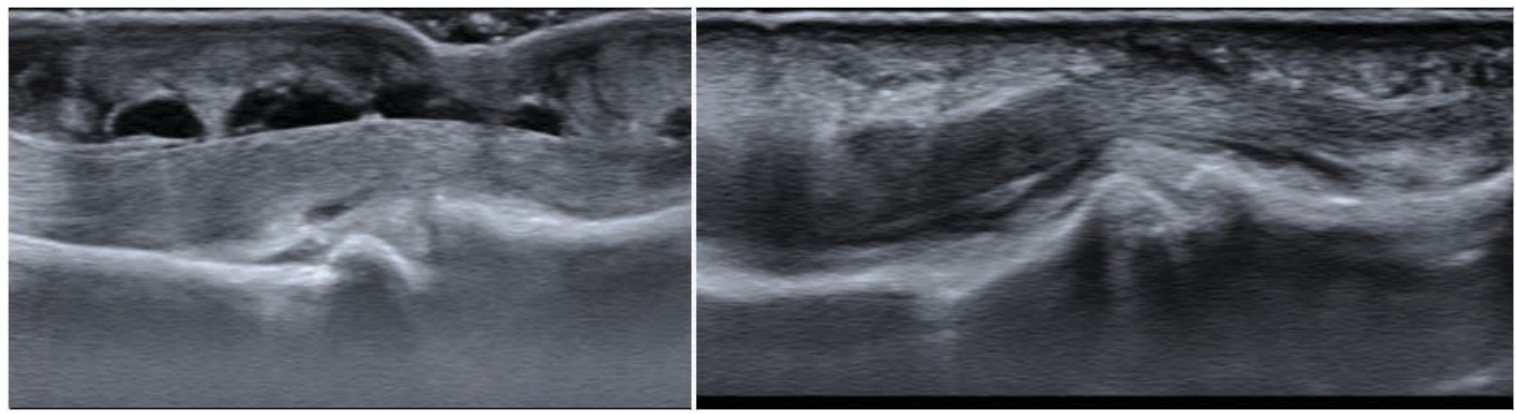

FIGURE 2. Palmar longitudinal scan of the 3rd and 4th finger showing tenosynovitis of the flexor tendon

of PD positivity at the wrists (11). Interestingly, there was a similar prevalence of joint effusion in rheumatoid arthritis (RA) patients, however, the presence of PD signal and synovial hypertrophy were more frequent in RA patients, especially PD grade 2 or $3(7,8)$.

\section{Tendons}

Tendon involvement is another common manifestation in SSc patients. Tenosynovitis of the hand was found in $27 \%$ of SSc patients, showing a double pattern, inflammatory and sclerosing type (8). The sclerosing pattern is defined as an iso- or hyperechoic hypertrophy of the tendon sheath with concentric alternating iso-and/or hyperechoic layers and it has been described as a specific characteristic of the disease $(8,12)$. An important thing is that sclerosing tenosynovitis is clinically characterized by palpable tendon friction rubs (13). Tendon friction rubs and also sclerosing tenosynovitis in the hand, has been demonstrated to be associated with diffuse cutaneous involvement and interstitial lung disease $(12,14)$. This specific pattern is more frequently detected at the extensor tendons of the hand (8). Another frequent manifestation in SSc patients detected by US in hands is A1 pulley thickening (15). This feature is not specific to SSc but it is correlated with hand disability and disease duration (16).

US enthesopathy are frequently found in SSc patients. Furthermore, common extensor tendons en- thesopathy was correlated with synovio-entheseal complex inflammation, suggesting that entheseal inflammation in SSc may share the same micro-anatomical targets as found in $\operatorname{SpA}(17)$.

\section{Bones}

Distinctive abnormalities detected by US include bone erosions, cortical irregularities, acroosteolysis and osteophytes. Erosions are small, discrete, and less invasive than those of RA (5). There are much less common than in RA patients being found in up to $11 \%$ of SSc cases (7). Cortical irregu $\neg$ larities are described as loss of continuity of cortical bone without any clear evidence of erosion/osteophyte/fracture. In some studies the prevalence of bone erosions and cortical irregularities was not signifi $\neg$ cantly higher in patients than in healthy controls (10).

The normal distal phalanx, when assessed with US via a dorsal approach, showed either a straight or slightly concave cortical hyperechoic line at its waist that ends with small elevation at the tuft. Disappearance of this outline was defined as a sign of acroosteolysis, as well as an abrupt ending of the dorsal cortex. The most of the patients present PD signal in the area of resorption. (18). This represent a characteristic feature of the disease and it was found in $20 \%$ of cases with a $90 \%$ sensitivity (18).

According to Keen et al. US osteophytes are defined as cortical protrusions seen in two planes 


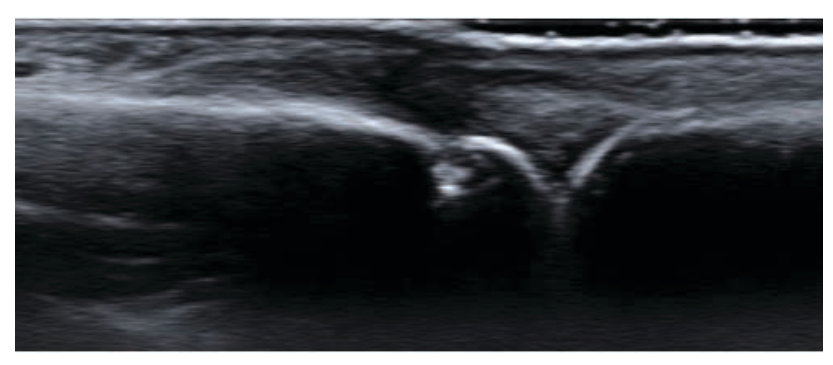

FIGURE 3. Lateral longitudinal scan of the $2^{\text {nd }}$ metacarpophalangeal joint showing a small erosion of $2^{\text {nd }}$ metacarpal head bone

$(19,20)$. This is a nonspecific finding and it appears in the context of degenerative lesions of the joint (15). US showed a significantly higher number of joints with osteophytes than X-rays (59 vs 27\%) (7).

\section{CALCINOSIS}

Calcinosis represent another specific characteristic of the disease and it is defined as hyperechoic foci with or without acoustic shadowing. In order of frequency it can be located in different sites - soft tissue (71\%), peritendinous $(47 \%)$, periarticular $(35 \%)$ and tendon (12\%) (18). Sensitivity of US in calcinosis detection was demonstrated to be up to $89 \%$ (18) but was discovered in varying percentages $27 \%-40 \%$ $(7,8,18)$. An interesting correlation between ulnar artery occlusion, a marker of decreased peripheral perfusion which will be discussed below, and X-ray identified calcinosis was recently found, claiming an association between vascular disease in $\mathrm{SSc}$ and the pathogenesis of calcinosis (21).

Calcinosis can determine the appearance of twinkling artifact (15). The twinkling artifact represent the presence of colour or power Doppler signal immediately behind a high echogenic area, such as urinary tract stones, soft tissue calcific deposits or bones, acquiring a false appearance of blood flow (22).

\section{NERVE}

Neurological complications occur between 1 and $40 \%$ of SSc patients (23). Ultrasonography is an easy method to detect different types of nerve lesions, such as nerve entrapments, nerve tumors, and traumatic nerve injuries. To identify carpal tunnel syndrome is very easy to evaluate the median nerve at carpal tunnel level by measuring the nerve cross-sectional area. Median nerve area and also transverse diameter are increased in all phases of asymptomatic SSc patients, independently to clinical variables (24). Another study shown that median nerve area was significantly different between the 3 phases of skin in- volvement, being higher in patients in the edematous phase (25).

US can be used for a quantitative evaluation of nerve density that can separate normal nerves from pathologic nerves (26). An automated software analyzes nerve images to calculates the density. In limited cutaneous SSc patients, median nerve density was reduced, especially in the symptomatic group, compared to control subjects. The nerve was evaluated outside carpal tunnel to avoid overlapping results with compressive neuropathies (27). The possible etiology of peripheral nerve damage in SSc patients could be a vascular or autoimmune dependent neuropathy with primary involvement of the vasa nervorum (28).

\section{VASCULARIZATION}

Raynaud phenomenon (RP) and vascular manifestations in SSc may be the primary events, even preceding skin involvement. Vascular involvement in SSc is characterized by two main mechanisms. The first is an early destructive vasculopathy with progressive loss of capillaries. The second is an obliterative vasculopathy due to intimal hyperplasia with proliferation of vascular cells and intimal fibrosis (29). US can be used in assessment and monitoring both of microvascular and macrovascular damages.

Regarding microvascular involvement Doppler US proved to be useful in distinguishing primary by secondary RP. The nail bed vascularity was analyzed before and after a cold and warm challenge. Patients with primary RP had normal vascularity at ambient temperature but differed from healthy controls in the response to a dynamic temperature challenge with a more pronounced decrease of signal after the cold test. In contrast to healthy controls and primary RP patients, secondary RP patients showed a lower vascularity also at ambient temperature and even a more pronounced difference was observed after the cold challenge. US was able to distinguish primary RP from secondary RP in up to $90 \%$ of the cases $(30,31)$. A good level of correlation was found between nailfold capillaroscopy and Power Doppler US findings both in primary and secondary RP (31).

At the hand level macrovascular involvement can be assessed by investigating hand and finger arteries using color Doppler. The US image of finger arteries in systemic sclerosis is entirely different from that in vasculitis. They appear very small with decreased pulsation and frequently with stenoses which lead to increased blood flow velocities and turbulent flow. 
Artery walls are thickened and slightly hyperechoic (32). Schmit et al. (33) evaluated the superficial palmar arch, and the radial and ulnar arteries at the wrists after an induced vasodilatation with hot water. Three different types of vascular pathology was described in this study: "type 1" showing narrowing or chronic occlusion of some proper digital arteries; "type 2" characterized by the same finding affecting all proper digital arteries; and "type 3" including acute occlusions. SSc patients mainly have vascular type 1 or 2 (33).

Ulnar artery occlusion (UAO) is considered as a frequent and specific vascular feature of SSc $(8,34,35)$. On the other hand, radial artery is rarely involved (36). UAO has been shown to associate with digital ulcers (DUs), pitting scars, calcinosis, and acroosteolysis (29) and it has proven to represent an important risk factor for the development of DUs in patients with SSc (37). Another recent multicenter study demonstrate that UAO can be a severity marker of vasculophaty in SSc and has shown an association of UAO with key severity markers of vasculopathy such as the late capillaroscopic pattern according to Cutolo's classification, the presence of skin telangiectasia, altered DLCO measures and the history of fingertip ischemic DUs (38). UAO and pathological finger pulp blood flow (FPBF) assessed by power doppler ultrasonography are associated with a severe capillary loss on capillaroscopic evaluation $(29,39)$ and also with a history of multiple episodes of ischemic DUs. Even more the association between UAO and pathological FPBF in the same patient is an independent predictor of new ischemic DUs (40). A pathological FPBF was defined as a significant decrease of the doppler signal on the peripheral part of the finger pulp or on the entire finger pulp recorded at least on one of the two evaluated fingers (40). DUs are also associated with digital artery resistivity index (39). The resistivity index, defined as the difference between the peak systolic and end diastolic flow velocities divided by the peak systolic velocity, was calculated at radial and ulnar proper palmar digital arteries at the level of the proximal phalanx of third and fourth fingers.

\section{SKIN}

The progressive skin fibrosis represent the clinical hallmark of the SSc and also a marker for disease classification and activity. Clinically it is used the modified Rodnan skin score (mRSS) to evaluate the severity of skin thickening (41). The mRSS has some disadvantages, as it is unable to detect small degrees of skin thickness, difficulty to differentiate between oedematous and fibrotic phase and a high variability among different examiners $(42,43)$. In recent years, high-frequency US has become a useful method in skin assessment by capacity to measure skin thickness and to depict accurately different layers of the skin providing a detailed image of the morphostructural changes. It has been shown to be a valid measurement of skin thickening with a high correlation to RSS (44) Cross sectional studies have shown excellent interand intraobserver variability for dermal thickness (4547). High-frequency US can separate epidermis, dermis and subcutaneous layers, can identify oedematous phase, which may precede palpable skin involvement and may thus be useful to early diagnosis and to identify patients with diffuse form very early in the disease process $(44,46)$. Subclinical dermal involvement may be also detectable by US even in skin areas with a normal mRSS (48). Further, dermal finger thickness proved to be associated with nailfold microangiopathy severity assessed by capillaroscopy (49).

A relatively new US technology to analyze elastic properties of tissues is US elastography (UE) and mainly includes strain elastography and shear wave elastography. The strain elastography examines the deformability of a given tissue during its controlled compression with an ultrasonographic transducer. The result is expressed on a semiquantative color scale or as a strain ratio obtained by dividing the deformability of the tissue of interest by the deformability of a prespecified reference area (50). However, this technique is qualitative rather than quantitative because of its operator dependence (51). On the other hand shear wave elastography examines the speed at which the transducer-generated wave is propagated across the examined tissue and it were been proven to be more accurate and reproducible than those obtained during strain elastography (50).

The first study using UE in SSc patients was made in 2010, after other studies demonstrated that UE can improve the measurement of dermal thickness and also the assessment of fibrotic skin (52). The studies using either strain elastography or shear wave elastography showed that the elastographic strain of the skin in SSc patients is higher than those of healthy controls and that elastographic parameters are characterized by a high repeatability and reproducibility (52-54). In last years shear wave elastography was much more studied in SSc and it proved to reflect the degree of skin involvement and to have a higher sensitivity in detecting subtle skin changes than US (55) and also than RSS, especially in cases with non-detectable 
changes at physical evaluation (56). This non-invasive, real-time and operator-independent imaging technique could be an ideal tool for the assessment of SSc disease (51).

\section{CONCLUSIONS}

Permanent improvement of US technology allowed new research in the field which led to multiple and more important applications of US in SSc. In rheumatology US become an extension of the clinical examination and particularly in SSc it helps to a bet-

Conflict of interest: none declared

Financial support: none declared

\section{REFERENCES}

1. Steen VD, Medsger TAJ. Severe organ involvement in systemic sclerosis with diffuse scleroderma. Arthritis Rheum. 2000 Nov;43(11):2437-44.

2. Gutierrez M, Pineda C, Cazenave T, et al. Ultrasound in systemic sclerosis. A multi-target approach from joint to lung. Clin Rheumatol. 2014;33(8):1039-47.

3. Avouac J, Clements PJ, Khanna D, et al. Articular involvement in systemic sclerosis. Rheumatology (Oxford). 2012 Aug;51(8):1347-56.

4. Varga J, Trojanowska M, Kuwana M. Pathogenesis of systemic sclerosis: recent insights of molecular and cellular mechanisms and therapeutic opportunities. J Scleroderma Relat Disord. 2017 Jul 19;2(3):137-52.

5. Morrisroe KB, Nikpour M, Proudman SM. Musculoskeletal Manifestations of Systemic Sclerosis. Rheum Dis Clin North Am. 2015;41(3):507-18.

6. Avouac J, Walker U, Tyndall A, et al. Characteristics of joint involvement and relationships with systemic inflammation in systemic sclerosis: results from the EULAR Scleroderma Trial and Research Group (EUSTAR) database. J Rheumatol. 2010 Jul;37(7):1488-501.

7. Cuomo G, Zappia M, Abignano G, et al. Ultrasonographic features of the hand and wrist in systemic sclerosis. Rheumatology (Oxford). 2009;48(11):1414-7.

8. Elhai M, Guerini $\mathrm{H}$, Bazeli R, et al. Ultrasonographic hand features in systemic sclerosis and correlates with clinical, biologic, and radiographic findings. Arthritis Care Res. 2012;64(8):1244-9.

9. Hubac J, Gilson M, Gaudin P, et al. Ultrasound prevalence of wrist, hand, ankle and foot synovitis and tenosynovitis in systemic sclerosis, and relationship with disease features and hand disability. Jt Bone Spine. 2020;87(3):229-33.

10. lagnocco A, Vavala $C$, Vasile $M$, et al. Power Doppler ultrasound of the hand and wrist joints in systemic sclerosis. Clin Exp Rheumatol. 2013;31(SUPPL.76).

11. Ruaro B, Sakellariou G, Cutolo M, et al. Well-trained sonographers are worth their weight in gold: ultrasound in systemic sclerosis. Rheumatology (Oxford). 2018;57(11):1883-4.

12. Avouac J, Walker UA, Hachulla E, et al. Joint and tendon involvement predict disease progression in systemic sclerosis: a EUSTAR prospective study. Ann Rheum Dis. 2016 Jan;75(1):103-9.

13. Cuomo G, Zappia M, ludici $M$, et al. The origin of tendon friction rubs in patients with systemic sclerosis: a sonographic explanation. Arthritis Rheum. 2012 Apr;64(4):1291-3.

14. Lescoat A, Ballerie A, Belhomme N, et al. Synovial involvement assessed by power Doppler ultra-sonography in systemic sclerosis: Results of a cross-sectional study. Rheumatol (United Kingdom). 2018;57(11):2012-21.

15. Tamas MM. Systemic sclerosis. In: Fodor D, editor. Musculoskeletal ultrasound Volum 2. Bucharest: Librex Publishing; 2018. p. 78-85.

16. Tagliafico A, Panico N, Serafini G, et al. The thickness of the A1 pulleys reflects the disability of hand mobility in scleroderma. A pilot study using high-frequency ultrasound. Eur $\mathrm{J}$ Radiol. 2011;77(2):254-7. ter evaluate of the skin, blood vessels, joints and tendons involvement. It also allows the differentiation between primary and secondary RP, between limited and diffuse form of the disease bringing new elements for a better understanding of SSc pathogenesis. All this making US to become an important tool in disease assessment.

\section{Acknowledgement}

All authors contributed to coordination and also they read and approved the final manuscript.

17. Terenzi R, Karalilova R, Lepri G, et al. Enthesopathy and involvement of synovio-entheseal complex in systemic sclerosis: an ultrasound pilot study. Rheumatology (Oxford). 2020 Mar;59(3):580-5.

18. Freire V, Bazeli R, Elhai M, et al. Hand and wrist involvement in systemic sclerosis: US features. Radiology. 2013;269(3):824-30.

19. Keen HI, Lavie F, Wakefield RJ, et al. The development of a preliminary ultrasonographic scoring system for features of hand osteoarthritis. Ann Rheum Dis. 2008 May;67(5):651-5.

20. Keen HI, Wakefield RJ, Grainger AJ, et al. Can ultrasonography improve on radiographic assessment in osteoarthritis of the hands? A comparison between radiographic and ultrasonographic detected pathology. Ann Rheum Dis. 2008 Aug;67(8):1116-20.

21. Fairchild $R$, Chung $M$, Sharpless $L$, et al. Ultrasound Detection of Calcinosis and Association with Ulnar Artery Occlusion in Patients with Systemic Sclerosis. Arthritis Care Res (Hoboken). 2020 May;

22. Pracoń G, Płaza M, Walentowska-Janowicz M, et al. The value of ultrasound in the diagnosis of limited scleroderma - a case report. Vol. 15, Journal of ultrasonography. 2015. p. 326-31.

23. Amaral TN, Peres FA, Lapa AT, Marques-Neto JF, Appenzeller S. Neurologic involvement in scleroderma: a systematic review. Semin Arthritis Rheum. 2013 Dec;43(3):335-47.

24. Bandinelli F, Kaloudi O, Candelieri A, et al. Early detection of median nerve syndrome at the carpal tunnel with high-resolution $18 \mathrm{MHz}$ ultrasonography in systemic sclerosis patients. Clin Exp Rheumatol. 2010;28(5 Suppl 62):S15-8.

25. Sousa-Neves J, Cerqueira M, Afonso C, et al. Median nerve ultrasound findings in systemic sclerosis patients: How do they relate to clinical features of the disease? Vol. 14, Reumatologia clinica. Spain; 2018. p. 312-3

26. Tagliafico A, Tagliafico G, Martinoli C. Nerve density: a new parameter to evaluate peripheral nerve pathology on ultrasound. Preliminary study. Ultrasound Med Biol. 2010 Oct;36(10):1588-93.

27. Bignotti B, Ghio M, Panico N, et al. High-resolution ultrasound of peripheral nerves in systemic sclerosis: a pilot study of computeraided quantitative assessment of nerve density. Skeletal Radiol. 2015;44(12):1761-7.

28. Tagliafico A, Panico N, Resmini E, et al. The role of ultrasound imaging in the evaluation of peripheral nerve in systemic sclerosis (scleroderma). Eur J Radiol. 2011 Mar;77(3):377-82.

29. Lescoat A, Coiffier G, de Carlan M, et al. Combination of Capillaroscopic and Ultrasonographic Evaluations in Systemic Sclerosis: Results of a Cross-Sectional Study. Arthritis Care Res. 2018;70(6):938-43.

30. Keberle M, Tony HP, Jahns R, et al. Assessment of microvascular changes in Raynaud's phenomenon and connective tissue disease using colour doppler ultrasound. Rheumatology (Oxford). 2000 Nov;39(11):1206-13.

31. Lee $\mathrm{S}$ II, Lee SY, Yoo WH. The usefulness of power Doppler ultrasonography in differentiating primary and secondary Raynaud's phenomenon. Clin Rheumatol. 2006 Nov;25(6):814-8.

32. Schmidt WA, Wernicke D, Kiefer E, et al. Colour duplex sonography of finger arteries in vasculitis and in systemic sclerosis. Ann Rheum Dis. 2006 Feb;65(2):265-7. 
33. Schmidt WA, Krause A, Schicke B, et al. Color Doppler ultrasonography of hand and finger arteries to differentiate primary from secondary forms of Raynaud's phenomenon. J Rheumatol. 2008 Aug;35(8):1591-8.

34. Taylor MH, McFadden JA, Bolster MB, et al. Ulnar artery involvement in systemic sclerosis (scleroderma). J Rheumatol. 2002 Jan;29(1):102-6.

35. Carpentier PH, Biro C, Jiguet M, et al. Prevalence, risk factors, and clinical correlates of ulnar artery occlusion in the general population. J Vasc Surg. 2009 Dec;50(6):1333-9.

36. Stafford L, Englert H, Gover J, et al. Distribution of macrovascular disease in scleroderma. Ann Rheum Dis. 1998 Aug;57(8):476-9

37. Frerix M, Stegbauer J, Dragun D, et al. Ulnar artery occlusion is predictive of digital ulcers in SSc: a duplex sonography study. Rheumatology (Oxford). 2012 Apr;51(4):735-42.

38. Lescoat A, Yelnik CM, Coiffier G, et al. Ulnar Artery Occlusion and Severity Markers of Vasculopathy in Systemic Sclerosis: A Multicenter Cross-Sectional Study. Arthritis Rheumatol (Hoboken, NJ). 2019 Jun;71(6):983-90.

39. Schioppo T, Orenti A, Boracchi P, et al. Evidence of macro- and micro-angiopathy in scleroderma: An integrated approach combining 22-MHz power Doppler ultrasonography and video-capillaroscopy. Microvasc Res. 2019 Mar;122:125-30.

40. Lescoat A, Coiffier G, Rouil A, et al. Vascular Evaluation of the Hand by Power Doppler Ultrasonography and New Predictive Markers of Ischemic Digital Ulcers in Systemic Sclerosis: Results of a Prospective Pilot Study. Arthritis Care Res (Hoboken). 2017 Apr;69(4):543-51.

41. Furst DE, Clements PJ, Steen VD, et al. The modified Rodnan skin score is an accurate reflection of skin biopsy thickness in systemic sclerosis. J Rheumatol. 1998 Jan;25(1):84-8.

42. Clements PJ, Lachenbruch PA, Ng SC, et al. Skin score. A semiquantitative measure of cutaneous involvement that improves prediction of prognosis in systemic sclerosis. Arthritis Rheum. 1990 Aug;33(8):1256-63

43. Czirják L, Nagy $Z$, Aringer $M$, et al. The EUSTAR model for teaching and implementing the modified Rodnan skin score in systemic sclerosis. Ann Rheum Dis. 2007 Jul;66(7):966-9.

44. Hesselstrand R, Scheja A, Wildt M, et al. High-frequency ultrasound of skin involvement in systemic sclerosis reflects oedema, extension and severity in early disease. Rheumatology (Oxford). 2008 Jan;47(1):84-7.
45. Moore TL, Lunt M, McManus B, et al. Seventeen-point dermal ultrasound scoring system--a reliable measure of skin thickness in patients with systemic sclerosis. Rheumatology (Oxford). 2003 Dec;42(12):1559-63.

46. Kaloudi O, Bandinelli F, Filippucci E, et al. High frequency ultrasound measurement of digital dermal thickness in systemic sclerosis. Ann Rheum Dis. 2010 Jun;69(6):1140-3.

47. Akesson A, Hesselstrand R, Scheja A, et al. Longitudinal development of skin involvement and reliability of high frequency ultrasound in systemic sclerosis. Ann Rheum Dis. 2004 Jul;63(7):791-6.

48. Sulli $A$, Ruaro $B$, Smith $V$, et al. Subclinical dermal involvement is detectable by high frequency ultrasound even in patients with limited cutaneous systemic sclerosis. Arthritis Res Ther. 2017 Mar;19(1):61.

49. Sulli A, Ruaro B, Alessandri E, et al. Correlations between nailfold microangiopathy severity, finger dermal thickness and fingertip blood perfusion in systemic sclerosis patients. Ann Rheum Dis. 2014 Jan;73(1):247-51.

50. Shiina T, Nightingale KR, Palmeri ML, et al. WFUMB guidelines and recommendations for clinical use of ultrasound elastography: Part 1: basic principles and terminology. Ultrasound Med Biol. 2015 May;41(5):1126-47.

51. Yang Y, Qiu L, Wang L, et al. Quantitative Assessment of Skin Stiffness Using Ultrasound Shear Wave Elastography in Systemic Sclerosis. Ultrasound Med Biol. 2019 Apr;45(4):902-12.

52. lagnocco A, Kaloudi O, Perella C, et al. Ultrasound elastography assessment of skin involvement in systemic sclerosis: lights and shadows. J Rheumatol. 2010 Aug;37(8):1688-91.

53. Lee SY, Cardones AR, Doherty J. Preliminary Results on the Feasibility of Using ARFI/SWEI to Assess Cutaneous Sclerotic Diseases. Ultrasound Med Biol. 2015 Nov;41(11):2806-19.

54. Cannaò PM, Vinci V, Caviggioli F, et al. Technical feasibility of realtime elastography to assess the peri-oral region in patients affected by systemic sclerosis. J Ultrasound. 2014 Dec;17(4):265-9.

55. Yang $Y$, Yan F, Wang L, et al. Quantification of skin stiffness in patients with systemic sclerosis using real-time shear wave elastography: a preliminary study. Clin Exp Rheumatol. 2018;36 Suppl 1(4):118-25.

56. Sobolewski P, Maślińska M, Zakrzewski J, et al. Applicability of shear wave elastography for the evaluation of skin strain in systemic sclerosis. Rheumatol Int. 2020 May;40(5):737-45. 